\title{
Männerpolitik und Antifeminismus
}

\author{
THOMAS GESTERKAMP
}

Mit dem Einzug der „Alternative für Deutschland” (AfD) in das Europaparlament und drei ostdeutsche Landtage etabliert sich nach dem Vorbild anderer europäischer Staaten eine rechtspopulistische Partei, zu deren Programmatik neben der Kritik am Euro und Ausländerfeindlichkeit auch der Kampf gegen die „Gender-Ideologie“ gehört. ${ }^{1}$ Die AfD beteiligt sich zum Beispiel an Demonstrationen und Kampagnen, die das Thema "sexuelle Vielfalt” in schulischen Bildungsplänen stoppen wollen. Sie wendet sich im Bündnis mit fundamentalistischen Christen gegen Abtreibung, propagiert in Wahlkämpfen wie etwa in Sachsen die traditionelle Kleinfamilie mit mindestens drei Kindern und macht Stimmung gegen die Frauenquote. Zulauf erhält die neue Partei auch von antifeministischen Männerrechtlern, die um ihre Privilegien fürchten und die althergebrachte männliche Herrschaft in den Geschlechterbeziehungen erhalten oder wiederherstellen wollen. Mit diesem veränderten Umfeld müssen sich gleichstellungspolitische Aktivitäten auseinandersetzen.

\section{Verschwörungstheorien}

Die Kernthese der Männerrechtler lautet: Die Gleichstellung der Geschlechter sei längst erreicht, die Emanzipation der Frauen abgeschlossen. Sie sprechen von einer „Kaste der Genderfunktionäre“, deren kulturelle Dominanz jeden Widerspruch unterdrücke. Mit der Realität hat diese Behauptung wenig zu tun. Denn wann immer sich die Medien in der Vergangenheit mit dem komplizierten Wortpaar Gender Mainstreaming beschäftigten, schwankte die Bewertung zwischen lächerlich und gefährlich.
Der Stern bezeichnete die „neue Geschlechtergefühligkeit“ schon vor über einem Jahrzehnt als „trivial und teuer “. ${ }^{3}$ Der Spiegel malte ein düsteres Bild autoritärer GenderPädagogik, das Jungen „früh zu Kritikern des eigenen Geschlechts" mache. ${ }^{4}$ Der Focus schrieb mit ständigen Berichten über das "geschwächte Geschlecht“ eine „neue Bürgerrechtsbewegung“ geradezu herbei. ${ }^{5}$ Die Frankfurter Allgemeine Zeitung wetterte gegen ein „angewandtes Kaderprinzip der feministischen Lobby“, die eine „politische Geschlechtsumwandlung “ plane. ${ }^{6}$ Die rechtskonservative Junge Freiheit nahm solche Verschwörungstheorien dankbar auf: Hier werde „eine totalitäre Ideologie durch eine auserwählte Truppe Linientreuer von oben nach unten durchgesetzt“.

Hauptbühne der Antifeministen sind aber nicht die Printmedien, sondern das Internet. Webseiten wie „wgvdl“ („Wieviel Gleichberechtigung verträgt das Land?“) oder das Forum Männerrechte, das dem Verein MannDat nahe steht, stilisieren Männer zu Diskriminierten in allen Lebenslagen. ${ }^{8}$ Der Tenor der Netzbeiträge schwankt zwischen Gekränktsein und verbaler Aggression: Andersdenkende Männer gelten als „Lila Pudel“, die dem Feminismus dienen. Inhaltlich überwiegen Zerrbilder und schlichte Erklärungen. Ein Beispiel aus dem Bereich der beruflichen Ungleichheit ist die Behauptung, die schlechtere Bezahlung von Frauen sei keine Diskriminierung. Das Ziel der Überwindung der Entgeltdifferenz wird delegitimiert: Männer verdienten zu Recht mehr, weil sie anstrengendere Berufe ausübten und durchgehend erwerbstätig seien. Frauen hätten nur deshalb Nachteile, weil sie sich freiwillig gegen eine geradlinige Karriere entschieden; Förderprogramme oder gar Quoten seien daher unnötig.
1 Vgl. Kemper, A. (2014): Keimzelle der Nation? Familienund geschlechterpolitische Positionen der AfD: FriedrichEbert-Stiftung, Berlin.

2 Paulwitz, M. (2008): Freiheit statt Feminismus, in: Junge Freiheit $7 / 2008$, S. 1.

3 Schneider, K. (2003): Ich Mann, du Frau, in: Stern 12/2003, S. 64.

4 Pfister; R. (2007): Der neue Mensch. Unter dem Begriff "Gender Mainstreaming" haben Politiker ein Erziehungsprogramm für Männer und Frauen gestartet, in: Der Spiegel 1/2007, S. 27.
5 Klonovsky, M. (2008): Das geschwächte Geschlecht. Emanzipation, nächste Stufe: Gegen die Benachteiligung und Abwertung von Männern formiert sich eine neue Bürgerrechtsbewegung, in: Focus 41/2008, S. 126.

6 Zastrow, V. (2006): Politische Geschlechtsumwandlung in: Frankfurter Allgemeine Zeitung vom 19. Juni, S. 8.

7 Paulwitz, M. (2008), a.a.o.

8 Vgl. ausführlich Rosenbrock, H. (2012): Die antifeministische Männerrechtsbewegung, Berlin. 


\section{Umkämpfte Begriffe}

Der „ausufernde Gouvernanten- und Umerziehungsstaat“, so heißt es, bevorzuge einseitig die Frauen. „Freiheit statt Feminismus!" fordert kurzerhand die Junge Freiheit, und prangert „Denkverbote“ an. ${ }^{9}$ Kritik an der Benachteiligung von Männern sei schlicht unerwünscht. Wie viele Rechtskonservative sonnen sich Männerrechtler darin, als Tabubrecher die "political correctness“ zu missachten. Emanzipatorische Begriffe wie „Befreiung“ oder „Geschlechterdemokratie" versuchen sie umzudefinieren. Der Online-Auftritt ,freiewelt.net", der der AfD nahesteht, trägt den harmlosen und irreführenden Untertitel „Die Internet\& Blogzeitung für die Zivilgesellschaft". Eine andere Publikation nennt sich „eigentümlich frei“ - die Macher verstehen sich als Libertäre, sprachlich wie personell aber gibt es Schnittmengen mit der Jungen Freiheit.

Eine wichtige Denkfigur ist der Hinweis auf die Biologie, der die von Bestsellern massenhaft verbreitete These „Männer sind vom Mars, Frauen von der Venus“ aufgreift. Einzelergebnisse aus Hirnforschung oder Verhaltensbiologie sollen fragwürdige Behauptungen zur Geschlechterdifferenz untermauern. Den Befürwortern des Gender Mainstreaming wird vorgehalten, eine , anthropologische Neutralisierung" anzustreben und wissenschaftliche Fakten zu bestreiten. Ein weiteres Kennzeichen ist der AntiEtatismus. Dieser wendet sich gegen eine „Bevormundung“ durch öffentliche Institutionen, die sich angeblich zu sehr in die Aufgabenteilung zwischen Mann und Frau einmischten. Das Muster ähnelt der Argumentation christlicher Fundamentalisten in der Debatte um Krippen und Kitas. Organisatorisch haben die beiden Strömungen nur wenig miteinander zu tun, gemeinsam aber ist ihnen das Schüren von Ressentiments gegen den Staat. ${ }^{10}$

\section{Viktimisierung}

Die Herausgeber des antifeministischen Sammelbands „Befreiungsbewegung für Männer “ fordern „das Ende des weiblichen Geschlechtermonologs". ${ }^{11}$ Die Kerngruppe der Autoren gründete den Verein Agens, die „Arbeitsgemeinschaft zur Verwirklichung der Geschlechter-Demokratie“. Als politische Plattform diente zunächst das „Berliner Manifest,“ ein dürftiges Positionspapier, das den „gemeinsamen Dialog auf Augenhöhe zwischen der befreiten Frau und dem befreiten Mann“ propagierte. Die Agens-Gruppe suchte den Kontakt zu anerkannten Experten. 2011 konnte sie im Wissenschaftszentrum Berlin eine Veranstaltung durchführen, auf der auch WZB-Chefin Jutta Allmendinger mitdiskutierte. Im Nachhinein beschwerte sich Agens über „Diskursverweigerung“, weil die antifeministischen Thesen im $\mathrm{Pu}$ blikum nicht gut angekommen waren. Die Reaktion kann als typisch gelten: Aus der gekränkten Haltung, man werde übersehen und ausgegrenzt, entsteht bei Antifeministen ein zusätzliches Element der Viktimisierung.
Wenig Berührungsängste zu Männerrechtlern hatten zeitweise einige Parteistiftungen. Eine Veranstaltung von Agens mit der FDP-nahen Friedrich-Naumann-Stiftung in Hannover scheiterte 2010 am fehlenden Publikumsinteresse. Die CDU-nahe Konrad-Adenauer-Stiftung führte 2009 die Tagung „Ein Männeraufbruch ist überfällig“ in Mainz durch. Eingeladen war eine Mischung aus rechtslastigen und moderaten Rednern: Das Spektrum reichte vom antifeministischen Blogger Arne Hoffmann über den zur Welt gewechselten Ex-taz-Redakteur Robin Alexander bis zu Hartmut Steeb, dem Generalsekretär der Deutschen Evangelischen Allianz - einer Dachorganisation pietistischer Gruppen, die am Wortlaut der christlichen Schöpfungslehre festhalten und Homosexualität für eine psychische Störung halten.

Als Netzkommentatoren nach den Attentaten von Oslo Sympathie für die nicht nur islamophoben, sondern auch antifeministischen Motive des Massenmörders Anders Breivik äußerten, führte dies zu einer Distanzierung gemäßigter Männerrechtler. Die Szene präsentiert sich seither heterogener, ist aber weitgehend isoliert und auf sich bezogen. ${ }^{12}$ Die selbst ernannte "Männerrechtsbewegung" hat ihren Zenit möglicherweise bereits überschritten. Das zeigt sich auch darin, dass das „Internationale Antifeminismustreffen“, zu dem (deutschsprachige) Sympathisanten zeitweise regelmäßig im Raum Zürich zusammengekommen waren, zuletzt nicht mehr stattgefunden hat. Auf der anderen Seite hat der Kampf gegen den „Gender-Wahn” in der AfD möglicherweise eine parteipolitische Heimat gefunden.

\section{Gegengewicht}

Emanzipatorische Männerpolitik, die ein Gegengewicht zu antifeministischen Strömungen bildet, ist in Deutschland seit 2010 institutionell organisiert. Das Bundesfamilienministerium richtete in der Zeit der schwarzgelben Koalition das Referat „Gleichstellungspolitik für Jungen und Männer“ ein. Als wichtigstes Ziel proklamierte die damalige Ministerin Kristina Schröder, Männern Chancen in pädagogischen und pflegerischen Berufen aufzuzeigen. Unter Rotgrün (1998-2005) und während der Großen Koalition (2005 - 2009) hatte es keine vergleichbare männerpolitische

9 Paulwitz, M. (2008), a.a.O.

10 Vgl. ausführlich Gesterkamp,Th. (2010): Geschlechterkampf von rechts - Wie Männerrechtler und Familienfundamentalisten sich gegen das Feindbild Feminismus radikalisieren: Friedrich-Ebert-Stiftung, WISO-Diskurs, Bonn.

11 Gruner, P. H./Kuhla, E. (Hrsg.) (2009): Befreiungsbewegung für Männer. Auf dem Weg zur Geschlechterdemokratie, Gießen, S. 9.

12 Vgl. ausführlich Kemper, A. (Hrsg.) (2012): Die Maskulisten. Organisierter Antifeminismus im deutschsprachigen Raum, Münster. 
Akzentsetzung gegeben. Parlamentarische Anfragen zum Thema Männer und Jungen stellten in dieser Zeitspanne die CDU-Fraktion 2004 und die FDP-Fraktion 2008, allerdings lag dem keine geschlechterpolitische Motivation zugrunde. Auslöser waren Interventionen der Arbeitgeberverbände, die sich nach dem PISA-Schock Sorgen um das Qualifikationsniveau männlicher Schulabgänger machten. Es drohe ein „männliches Proletariat“, warnte der Deutsche Industrie- und Handelskammertag 2004; ähnlich argumentierte 2009 ein Gutachten des Aktionsrates Bildung im Auftrag der Vereinigung der Bayerischen Wirtschaft.

Die Profilierungsversuche der früheren CDU-Ministerin Schröder („Jetzt sind die Männer dran“) schürten bei der damaligen Opposition Misstrauen. Als warnendes Exempel diente zudem Österreich: Dort hatte eine schwarzbraune Regierung 2001 auf Initiative der rechtspopulistischen FPÖ und gegen den massiven Widerstand von Frauenverbänden eine „Männerpolitische Grundsatzabteilung“ im Sozialministerium etabliert. Einige (nicht alle) Publikationen, die die Abteilung in hohen Auflagen verbreitete, hatten eine betont konservative oder gar antifeministische Schlagseite. Die einst extrem polarisierte geschlechterpolitische Debatte im Nachbarland hat sich aber inzwischen deutlich entspannt. ${ }^{13}$

Was bedeutet das Aufkommen einer sich "freiheitlich“ gebenden, im Gedankengut aber eher rückwärts gewandten Ideologie für die Zukunft emanzipatorisch ausgerichteter Männerpolitik? Wie gefährlich ist die selbst ernannte „Männerrechtsbewegung “ für das Ziel der Geschlechtergerechtigkeit? Die Idee der Institutionalisierung durch ein Männerreferat ist nicht grundsätzlich falsch, nur weil eine umstrittene Ministerin sie in die Wege geleitet hat. Einige der von Antifeministen in überzogener Weise skandalisierten Themen benennen wichtige gesellschaftliche Probleme. So haben Jungen aus bildungsfernen Schichten mehr Schwierigkeiten in der Schule als Mädchen aus ähnlichem Milieu. Erst in jüngster Zeit gibt es für Männer eine geschlechtsspezifische und vom Staat geförderte Gesundheitsberichterstattung. Dass Gewalt nicht nur von Männern ausgeht, sondern sich im öffentlich Raum auch überwiegend gegen sie richtet, war lange Zeit ein unterbelichtetes Thema. Und so manchem Trennungsvater wird in der Tat übel mitgespielt, Kinder werden zum Faustpfand in Beziehungskonflikten instrumentalisiert. Nur lässt sich daraus bei aller Brisanz im Einzelfall keine flächendeckende gesellschaftliche Diskriminierung des Mannes qua Geschlecht ableiten. ${ }^{14}$

13 Vgl. Senk, J. (Hrsg.) (2013): Zwischen Gleichstellungserfolgen und Antifeminismus. Zwiespältige Tendenzen in der Modernisierung der Geschlechterverhältnisse, Linzer Schriften zu Gender und Recht, Bd. 52, Linz.

14 Vgl. Gesterkamp,Th. (2014): Jenseits von Feminismus und Antifeminismus - Plädoyer für eine eigenständige Männerpolitik, Heidelberg.

\section{Eigenständige Männerpolitik}

Ein Verbündeter im gemeinsamen Streiten von Frauen und Männern für Geschlechtergerechtigkeit ist das 2010 gegründete „Bundesforum Männer”, an dem neben der kirchlichen Männerarbeit und Sozialverbänden auch Jungenprojekte, Väterinitiativen und Schwulengruppen beteiligt sind. Der Zusammenschluss von mittlerweile über 30 Organisationen versteht sich als Lobby, Beratungsinstanz und Sprachrohr. In zehn Grundsätzen wird der „konstruktive Dialog zwischen den Geschlechtern“ befürwortet. Männliche Anliegen müssten in Ministerien, Behörden und Institutionen mehr Beachtung finden, fordert das Bundesforum. Von antifeministischen Strömungen hat sich der Dachverband ausdrücklich distanziert und lehnt die Kooperation mit Männerrechtler-Vereinen wie MannDat oder Agens ab.

An dem männerpolitischen Referat in ihrem Haus hält auch die derzeitige Frauen- und Familienministerin Manuela Schwesig (SPD) fest. Dort bieten sich institutionelle Möglichkeiten des Austausches zwischen weiblichen Akteurinnen und männlichen Akteuren. In der geschlechterpolitischen Debatte bleiben Männerthemen aber weiterhin randständig. Das gilt für die Europäische Union, die Gender Mainstreaming nach wie vor mit Frauenpolitik gleichsetzt, aber ebenso für den Ersten Gleichstellungsbericht der Bundesregierung, der vorrangig eine Perspektive auf den weiblichen Lebensverlauf einnahm.

Männerpolitik, das ist ein zentrales Anliegen der nicht antifeministisch orientierten Aktivisten, hat eine eigenständige Legitimation und sollte kein bloßes Anhängsel von Frauenpolitik sein. Eine durchaus parteiliche, aber dennoch dialogische Orientierung nimmt konfrontativen Antifeministen den Wind aus den Segeln. Der Gestus des Tabubrechers, der angebliche Denkverbote missachtet, wird ebenso erschwert wie das Umdeuten von Begriffen wie Befreiung, Zivilgesellschaft oder Geschlechterdemokratie. Antifeministische Männerrechtler bleiben trotz der AfD-Wahlerfolge bisher eine vorwiegend im Internet auffällige Minderheit - und bilden keineswegs die Mehrheit der „Männerbewegung“ in Deutschland.

\section{AUTOR}

THOMAS GESTERKAMP, Dr., promovierte in Politikwissenschaft über "Männliche Arbeits- und Lebensstile in der Informationsgesellschaft". Er ist Journalist und Autor zahlreicher Bücher, zuletzt „Jenseits von Feminismus und Antifeminismus" (2014). Bei der Friedrich-Ebert-Stiftung erschien seine Expertise "Geschlechterkampf von rechts - Wie Männerrechtler und Familienfundamentalisten sich gegen das Feindbild Feminismus radikalisieren."

thomas.gesterkamp@t-online.de 